\title{
Efecto de dos niveles de harina de laritaco (Vernonanthura patens) sobre la respuesta productiva y morfometría intestinal en pollos de engorde
}

\author{
Effect of two levels of «laritaco» meal (Vernonanthura patens) on the productive \\ performance and intestinal morphometry in broilers
}

\author{
Guido Apolo Arévalo ${ }^{1,3}$, Diego Rodríguez Saldaña²
}

\section{Resumien}

El objetivo del estudio fue evaluar el efecto de dos niveles ( 0.5 y 1\%) de inclusión de harina de laritaco (Vernonanthura patens) en el comportamiento productivo y morfometría intestinal de pollos de engorde. Se utilizaron 588 pollitos machos de la línea Cobb 500, distribuidos en un diseño de bloques en tres tratamientos con siete repeticiones cada uno, considerándose como unidad experimental un grupo de 28 pollos. No hubo diferencias entre tratamientos en consumo de alimento, peso corporal y conversión alimenticia; sin embargo, se observó aumento en la longitud de los segmentos del intestino, así como vellosidades intestinales más anchas y criptas menos profundas.

Palabras clave: morfometría, fitobióticos, vellosidad intestinal, criptas de Lieberkühn

\section{Abstract}

The aim of this study was to evaluate the effect of two levels $(0.5$ and $1 \%)$ of inclusion of «laritaco» meal (Vernonanthura patens) on the productive behavior and intestinal morphometry of broilers. In total, 588 male chicks of the Cobb 500 line were used, distributed in a block design in three treatments with seven repetitions each, considering a group of 28 chickens as an experimental unit. There were no differences between treatments in feed consumption, body weight and feed conversion; however, an increase in the length of the intestine segments was observed, as well as wider intestinal villi and shallower crypts.

Key words: morphometry, phytobiotics, intestinal villi, Lieberkühn crypts

\footnotetext{
${ }^{1}$ Universidad de las Fuerzas Armadas, Sangolquí, Ecuador

${ }^{2}$ Carrera de Medicina Veterinaria, Facultad de Ciencias Agropecuarias, Universidad de Cuenca, Ecuador

${ }^{3}$ E-mail: apolo.guido@hotmail.com
}

Recibido: 29 de julio de 2020

Aceptado para publicación: 28 de enero de 2021

Publicado: 24 de abril de 2021 


\section{INTRODUCCIÓN}

El desarrollo de un tracto gastrointestinal saludable es un elemento clave en la producción del pollo de engorde, ya que influye directamente en su desempeño productivo (Barrera et al., 2014). El epitelio intestinal facilita un flujo controlado y selectivo de componentes entre la luz y la mucosa; sin embargo está constantemente expuesto a altos niveles de antígenos bacterianos (Plaza et al., 2014), debiendo actuar como una barrera natural frente a las bacterias patógenas y sustancias tóxicas presentes en el alimento y que pueden alterar la microbiota y el epitelio, afectando la permeabilidad y causar procesos inflamatorios (Chávez et al., 2016). La capacidad del intestino para digerir y absorber el alimento está relacionada con la longitud y tamaño del intestino, así como con la densidad y disposición de las vellosidades intestinales (Jaramillo, 2012; Ordoñez et al., 2018).

Los aditivos fitogénicos o fitobióticos son productos naturales derivados de plantas que se usan en la alimentación animal para mejorar su rendimiento productivo (Roofc-haee et al., 2011). En este contexto, se destaca la importancia de buscar alternativas que representen una disminución al uso de aditivos sintéticos y que permitan disponer de productos naturales con actividad en la salud y la expresión productiva de los animales, como es el uso de plantas medicinales.

Diversas especies vegetales pueden ser empleadas como fuente de nutrientes o como aditivos medicinales. Una de las especies que se ha usado en Ecuador desde hace muchos años como medicina tradicional es el arbusto conocido como laritaco (Vernonant-hura patens), mencionándose su uso en el tratamiento de leishmaniasis (Gachet et al., 2010), así como para lavar heridas, tratar el dolor de cabeza y como cicatrizante (Kvist et al., 2006). También se ha indicado para el alivio de malestares estomacales, dolores de parto y para erupciones de la piel (Manzano et al., 2013).
El presente estudio tuvo como objetivo evaluar el efecto de la inclusión de dos niveles de harina de laritaco sobre la respuesta productiva y la morfometría intestinal de pollos de engorde, a fin de determinar su potencial uso como aditivo en la dieta.

\section{Materiales y MéTodos}

\section{Localización del Estudio}

La elaboración de la harina de laritaco así como la crianza de las aves se llevó a cabo en la granja «El Abuelo», ubicada en la parroquia Bellamaría del cantón Balsas, al sur de la provincia de El Oro. La zona se encuentra a una altitud de $600 \mathrm{msnm}$, con temperatura ambiental de $19-28{ }^{\circ} \mathrm{C}$. El análisis de las muestras histológicas se realizó en el Laboratorio ANIMALAB de la ciudad de Machachi. El trabajo se realizó durante los meses de julio y septiembre de 2017.

\section{Aves}

Se utilizaron 588 pollitos machos de la línea Cobb 500, distribuidos en un diseño de bloques completos al azar con tres tratamientos y siete repeticiones por tratamiento. La unidad experimental fue un grupo de 28 pollos alojados en divisiones de $3 \times 1.09 \mathrm{~m}$, dando una densidad de 8.56 pollos por metro cuadrado. Para el análisis estadístico se utilizó el modelo Proc Mixed con medidas repetidas del programa Statistical Analysis System (SAS) para medir ensayos cuando las observaciones son evaluadas sobre el mismo individuo en diferentes momentos de su desarrollo (medidas repetidas).

\section{Tratamientos}

Para evaluar el efecto de la inclusión de harina de laritaco en el rendimiento de los pollos broiler, se evaluaron tres tratamientos:

- T1: Control $(0 \%)$

- T2: Harina de laritaco $5 \mathrm{~kg} / \mathrm{t}(0.5 \%)$

- T3: Harina de laritaco $10 \mathrm{~kg} / \mathrm{t}$ (1\%) 


\section{Harina de Laritaco y Dietas}

Para la elaboración de la harina, se escogieron y recogieron en forma manual hojas frescas de plantas sanas de laritaco (Vernonanthura patens). Se colocaron en tendales de malla para un pre-secado por acción natural durante $72 \mathrm{~h}$, luego se sometieron a secado en un deshidratador de alimentos a $45^{\circ} \mathrm{C}$ durante $8 \mathrm{~h}$. Las hojas secas fueron trituradas en un molino manual hasta la obtención de harina, la cual se almacenó en un recipiente plástico hasta el momento de la elaboración de las dietas.

Las dietas fueron formuladas en base a maíz, pasta de soya y materia prima disponible en la zona (Cuadro 1), siguiendo las recomendaciones nutricionales de la línea genética Cobb-Vantress (2015) en tres fases: iniciación (1-10 días), crecimiento (11-22 días) y finalización (23-42 días). Todas las dietas estuvieron libres de antibióticos promotores del crecimiento (APC), y fueron iso proteicas, iso energéticas e iso fosfóricas (Cuadro 2), difiriendo entre sí únicamente por el nivel de inclusión de harina de laritaco $(0,0.5$ y $1 \%$ para $\mathrm{T} 1, \mathrm{~T} 2$ y $\mathrm{T} 3$ respectivamente).

\section{Parámetros Productivos}

El consumo de alimento por pollo se evidenció mediante el pesaje diario de la cantidad ofrecida menos la cantidad sobrante, dividida entre el número de aves existentes de cada repetición para obtener el consumo de alimento diario y semanal.

Al inicio del ensayo se pesaron las aves (peso inicial) con una balanza digital $( \pm 1 \mathrm{~g})$. Luego se pesaron los días 7, 14, 21, 28, 35 y 42. La ganancia de peso se obtuvo por diferencia entre los pesos semanales. El pesaje se hizo en forma grupal, pesando toda la unidad experimental hasta la tercera semana, y a partir del día 28 se realizaron los pesajes dividiendo la unidad experimental en tres subgrupos. La conversión alimenticia se comprobó mediante la relación entre el alimento consumido y el incremento de peso (consumo de alimento/incremento de peso).

\section{Morfometría del Paquete Visceral}

A los 21 y 42 días de edad se sacrificó al azar un pollo (por dislocación craneocervical) por repetición. Se tomó el paquete visceral, se vació su contenido y se registró el peso (g) del hígado, corazón, esófago-buche, proventrículo, molleja y se determinó la longitud $(\mathrm{cm})$ del intestino delgado, intestino grueso y ciego.

\section{Vellosidades Intestinales y Criptas de Lieberkühn}

Para el análisis histológico, en los animales sacrificados a los 42 días para las mediciones del paquete visceral, se tomó de cada ave (una por repetición) una porción de tres $\mathrm{cm}$ del asa duodenal, se lavó con agua destilada para eliminar el contenido, y se colocó en envases estériles con formol al 10\%. Las muestras se remitieron al laboratorio. Con el apoyo de un microscopio óptico con ocular micrométrico se determinó la altura y ancho de las vellosidades intestinales y la profundidad de las criptas de Lieberkühn (mm). La altura de vellosidad se tomó como la distancia que existe desde la porción superior de las criptas hasta el ápice de la vellosidad; el grosor de las vellosidades fue medido en el punto medio de la altura de estas y para la profundidad de cripta se consideró la distancia tomada desde la región basal de cada vellosidad hasta la parte basal superior de la musculatura lisa del intestino (Zea y Vílchez, 2014).

\section{Análisis Estadístico}

El modelo consideró como efectos fijos las variables discretas e independientes del tratamiento y los periodos de evaluación, así como las interacciones entre estos dos factores. Como variables dependientes se evaluaron los indicadores del desempeño general desde el día 0 hasta los 42 días: peso vivo, consumo de alimento y conversión alimenticia.

Para el caso del faenado se realizaron mediciones a los 22 y a los 42 días, coincidiendo con la finalización del experimento, 
Cuadro 1. Composición (\%) de las dietas utilizadas durante el ensayo

\begin{tabular}{|c|c|c|c|c|c|c|c|c|c|}
\hline \multirow{2}{*}{ Producto } & \multicolumn{3}{|c|}{ Inicial } & \multicolumn{3}{|c|}{ Crecimiento } & \multicolumn{3}{|c|}{ Finalizador } \\
\hline & $\mathrm{T} 1$ & $\mathrm{~T} 2$ & T3 & $\mathrm{T} 1$ & $\mathrm{~T} 2$ & T3 & $\mathrm{T} 1$ & $\mathrm{~T} 2$ & T3 \\
\hline Maíz & 59.9 & 59 & 59.2 & 60.5 & 59.4 & 59.4 & 61.61 & 60.68 & 60.77 \\
\hline Salvado de trigo & 3.1 & 3 & 3 & 3 & 3 & 3 & 3.15 & 2.91 & 2.505 \\
\hline Pasta de soya (47\%) & 29 & 29.4 & 28.8 & 29.3 & 29.4 & 28.8 & 27.2 & 27.5 & 27.1 \\
\hline Aceite de palma & 3.3 & 3.4 & 3.3 & 3.05 & 3.45 & 3.52 & 4.291 & 4.63 & 4.8 \\
\hline Carbonato de calcio & 1.2 & 1.2 & 1.18 & 1.1 & 1.1 & 1.1 & 0.955 & 0.95 & 0.97 \\
\hline Fosfato monobicálcico & 1.05 & 1.1 & 1.1 & 1 & 1.09 & 1.09 & 0.768 & 0.78 & 0.78 \\
\hline Bicarbonato de sodio & 0.35 & 0.31 & 0.32 & 0.3 & 0.3 & 0.3 & 0.207 & 0.21 & 0.21 \\
\hline Sal común & 0.35 & 0.35 & 0.35 & 0.3 & 0.3 & 0.3 & 0.335 & 0.34 & 0.34 \\
\hline Sulfato de cobre & 0 & 0 & 0 & 0 & 0 & 0 & 0.01 & 0.01 & 0.01 \\
\hline Enzima Fitasa & 0.01 & 0.01 & 0.01 & 0.01 & 0.01 & 0.01 & 0.01 & 0.01 & 0.01 \\
\hline $\begin{array}{l}\text { Enzima proteasa- } \\
\text { celulasa }\end{array}$ & 0.05 & 0.05 & 0.05 & 0.05 & 0.05 & 0.05 & 0.05 & 0.05 & 0.05 \\
\hline Premezcla Vitamínica & 0.25 & 0.25 & 0.25 & 0.25 & 0.25 & 0.25 & 0.26 & 0.26 & 0.26 \\
\hline DL-Metionina & 0.2 & 0.2 & 0.2 & 0.17 & 0.19 & 0.2 & 0.143 & 0.145 & 0.15 \\
\hline L-Lisina (Lisina $\mathrm{HCl}$ ) & 0.37 & 0.36 & 0.37 & 0.23 & 0.22 & 0.24 & 0.162 & 0.165 & 0.175 \\
\hline L-Treonina & 0.2 & 0.2 & 0.2 & 0.1 & 0.1 & 0.1 & 0.079 & 0.08 & 0.09 \\
\hline Cloruro de colina & 0.09 & 0.09 & 0.09 & 0.05 & 0.05 & 0.05 & 0.04 & 0.04 & 0.04 \\
\hline Antioxidante & 0.02 & 0.02 & 0.02 & 0.02 & 0.02 & 0.02 & 0.02 & 0.02 & 0.02 \\
\hline $\begin{array}{l}\text { Secuestrante de } \\
\text { Micotoxinas }\end{array}$ & 0.2 & 0.2 & 0.2 & 0.2 & 0.2 & 0.2 & 0.2 & 0.2 & 0.2 \\
\hline $\begin{array}{l}\text { Secuestrante de } \\
\text { Aflatoxinas }\end{array}$ & 0.1 & 0.1 & 0.1 & 0.1 & 0.1 & 0.1 & 0.01 & 0.1 & 0.1 \\
\hline Inhibidor de hongos & 0.2 & 0.2 & 0.2 & 0.2 & 0.2 & 0.2 & 0.2 & 0.2 & 0.2 \\
\hline Anticoccidial $^{1}$ & 0.06 & 0.06 & 0.06 & 0.07 & 0.07 & 0.07 & 0.07 & 0.07 & 0.07 \\
\hline $\begin{array}{l}\text { Pigmento (Xantófilas } \\
2 \% \text { ) }\end{array}$ & 0 & 0 & 0 & 0 & 0 & 0 & 0.15 & 0.15 & 0.15 \\
\hline Harina de laritaco & 0 & 0.5 & 1 & 0 & 0.5 & 1 & 0 & 0.5 & 1 \\
\hline Total & 100 & 100 & 100 & 100 & 100 & 100 & 100 & 100 & 100 \\
\hline
\end{tabular}

${ }^{1} \mathrm{El}$ anticoccidial se retiró la última semana del ensayo

T1: Control (0\%); T2: Harina de laritaco 5 kg/t (0.5\%); T3: Harina de laritaco 10 kg/t (1\%)

donde se determinó el peso vivo, así como del hígado, corazón, segmento esófago-buche, proventrículo y molleja (expresados en gramos) y la longitud $(\mathrm{cm})$ del intestino delgado, intestino grueso y ciego.
El modelo matemático utilizado que explica el comportamiento de las variables del desempeño correspondió a: $\mathrm{Y}_{\mathrm{ij} \mathrm{k}}=\mu+\mathrm{T}_{\mathrm{i}}+\mathrm{P}_{\mathrm{j}}$ $+\mathrm{TP}_{(\mathrm{ij})}+\varepsilon_{\mathrm{ijk}}$, donde $\mathrm{Y}_{\mathrm{j} \mathrm{k}}=$ variable respuesta (peso vivo; consumo, y conversión); $\mu=$ Media 
Cuadro 2. Valor nutricional (estimado) de las dietas

\begin{tabular}{lccccccccc}
\hline & \multicolumn{3}{c}{ Inicial } & \multicolumn{3}{c}{ Crecimiento } & \multicolumn{3}{c}{ Finalizador } \\
\cline { 2 - 10 } & T1 & T2 & T3 & T1 & T2 & T3 & T1 & T2 & T3 \\
\hline EM (kcal/kg) & 3095 & 3081 & 3067 & 3089 & 3088 & 3081 & 3172 & 3172 & 3172 \\
PB (\%) & 20.1 & 20.2 & 20.0 & 20.1 & 20.0 & 19.9 & 19.2 & 19.2 & 19.0 \\
Na (\%) & 0.24 & 0.24 & 0.23 & 0.21 & 0.21 & 0.21 & 0.20 & 0.20 & 0.20 \\
Ca (\%) & 0.91 & 0.92 & 0.91 & 0.87 & 0.88 & 0.88 & 0.78 & 0.78 & 0.78 \\
P Disp. (\%) & 0.45 & 0.46 & 0.46 & 0.44 & 0.46 & 0.46 & 0.40 & 0.40 & 0.40 \\
Lys (\%) & 1.18 & 1.18 & 1.18 & 1.09 & 1.09 & 1.08 & 1.00 & 1.00 & 1.00 \\
Met (\%) & 0.47 & 0.47 & 0.46 & 0.44 & 0.46 & 0.46 & 0.41 & 0.40 & 0.41 \\
\hline
\end{tabular}

T1: Control (0\%); T2: Harina de laritaco 5 kg/t (0.5\%); T3: Harina de laritaco 10 kg/t (1\%)

general de las observaciones; $\mathrm{T}_{\mathrm{i}}=$ efecto del $\mathrm{i}$ ésimo Tratamiento ( $\mathrm{i}=1,2,3) ; \mathrm{P}_{\mathrm{j}}=$ efecto del $\mathrm{J}$ ésimo periodo de evaluación $(\mathrm{J}=1,2 \ldots . .6$ semanas); $\mathrm{TP}=$ efecto de las interacción entre los factores principales (Tratamiento y Periodo); $\varepsilon_{\mathrm{ijk}}=$ error experimental, asumido normal e independientemente distribuido con media cero y varianza ó ${ }^{2} \mathrm{DNI} \sim\left(0, \sigma^{2}\right)$ (bajo el análisis por el GLM tradicional).

El modelo matemático utilizado que explica el comportamiento de las variables del faenado correspondió a: $\mathrm{Y}_{\mathrm{ijk}}=\mu+\mathrm{T}_{\mathrm{i}}+\mathrm{P}_{\mathrm{j}}+$ $\mathrm{TP}_{(\mathrm{ij})}+\varepsilon_{\mathrm{ijk}}$, donde $\mathrm{Y}_{\mathrm{ijk}}=$ variable respuesta (peso vivo y pesos del hígado, corazón, esófago-buche, proventrículo, molleja y la longitud del proventrículo, intestino delgado, intestino grueso y ciego); $\mu=$ Media general de las observaciones; $\mathrm{T}_{\mathrm{i}}=$ efecto del $\mathrm{i}_{\text {ésimo }}$ Tratamiento $(\mathrm{i}=1,2,3) ; \mathrm{Pj}=$ efecto del $\mathrm{J}$ período de evaluación $(\mathrm{J}=1,2) ; \mathrm{TP}=$ efecto de las interacción entre los factores principales (Tratamiento y Periodo); $\varepsilon_{\mathrm{ijk}}=$ error experimental, asumido normal e independientemente distribuido con media cero y varianza $\sigma^{2} \mathrm{DNI} \sim\left(0, \sigma^{2}\right)$ (bajo el análisis por el GLM tradicional).
El modelo matemático utilizado para explicar el comportamiento de las variables morfometría de las vellosidades intestinales y criptas de Lieberkühn correspondió a: $\mathrm{Y}_{\mathrm{ij}}=$ $\mu+\mathrm{T}_{\mathrm{i}}+\varepsilon_{\mathrm{ij}}$, donde $\mathrm{Y}_{\mathrm{ij}}=$ variable respuesta (altura y ancho de las vellosidades, y profundidad de las criptas); $\mu=$ Media general de las observaciones; $\mathrm{T}_{\mathrm{i}}=$ efecto del $\mathrm{i}_{-}{ }_{\text {ésimo }}$ Tratamiento $(\mathrm{i}=1,2,3) ; \varepsilon_{\mathrm{ij}}=$ error experimental, asumido normal e independientemente distribuido con media cero y varianza $\sigma^{2} \mathrm{DNI} \sim\left(0, \sigma^{2}\right)$ (bajo el análisis por el GLM tradicional).

Se consideraron significativos los valores de $\mathrm{p}<0.05$. En los casos donde se detectaron diferencias significativas entre los factores, se utilizó la instrucción LSMEANS para efectuar comparaciones de medias mediante prueba de $\mathrm{t}$ (SAS).

\section{Resultados y Discusión}

\section{Parámetros Productivos}

El consumo de alimento, peso corporal y conversión alimenticia al final del estudio (42 días no difirió entre tratamientos (Cuadro 3), lo 
Cuadro 3. Efecto de la inclusión de harina de laritaco ${ }^{1}$ (Vernonanthura patens) en la dieta sobre el comportamiento productivo en pollos broiler a los 42 días de edad $(\mathrm{n}=588$ pollos $)$

\begin{tabular}{lccc}
\hline Variable & $\mathrm{T} 1$ & $\mathrm{~T} 2$ & $\mathrm{~T} 3$ \\
\hline $\begin{array}{l}\text { Peso corporal } \\
\mathrm{g})\end{array}$ & $2688.99 \pm 28.78^{\mathrm{a}}$ & $2713.67 \pm 28.78^{\mathrm{a}}$ & $2706.44 \pm 28.78^{\mathrm{a}}$ \\
$\begin{array}{l}\text { Consumo de } \\
\text { alimento }(\mathrm{g})\end{array}$ & $4833.49 \pm 48.73^{\mathrm{a}}$ & $4887.83 \pm 48.73^{\mathrm{a}}$ & $4866.40 \pm 48.73^{\mathrm{a}}$ \\
$\begin{array}{l}\text { Conversión } \\
\text { alimenticia }\end{array}$ & $1.7987 \pm 0.019^{\mathrm{a}}$ & $1.8021 \pm 0.019^{\mathrm{a}}$ & $1.7979 \pm 0.019^{\mathrm{a}}$ \\
\hline
\end{tabular}

T1: Control (0\%); T2: Harina de laritaco 5 kg/t (0.5\%); T3: Harina de laritaco $10 \mathrm{~kg} / \mathrm{t}$ (1\%)

Cuadro 4. Efecto de la inclusión de harina de laritaco (Vernonanthura patens) en la dieta sobre el peso de los órganos del paquete visceral $(\mathrm{g})$ de pollos broiler a los 22 y 42 días de edad ( $\mathrm{n}=7$ pollos por tratamiento)

\begin{tabular}{lcccc}
\hline $\begin{array}{l}\text { Órganos } \\
(\mathrm{g})\end{array}$ & $\begin{array}{l}\text { Edad } \\
(\text { días })\end{array}$ & T1 & T2 & T3 \\
\hline Hígado & 22 & $27.57 \pm 1.84^{\mathrm{a}}$ & $26.71 \pm 1.84^{\mathrm{a}}$ & $28.14 \pm 1.84^{\mathrm{a}}$ \\
& 42 & $51.14 \pm 1.84^{\mathrm{a}}$ & $55.28 \pm 1.84^{\mathrm{a}}$ & $56.71 \pm 1.84^{\mathrm{a}}$ \\
Corazón & 22 & $6.14 \pm 0.30^{\mathrm{a}}$ & $5.57 \pm 0.30^{\mathrm{a}}$ & $5.85 \pm 0.30^{\mathrm{a}}$ \\
& 42 & $11.57 \pm 0.30^{\mathrm{a}}$ & $12.43 \pm 0.30^{\mathrm{a}}$ & $11.71 \pm 0.30^{\mathrm{a}}$ \\
Esófago-buche & 22 & $5.00 \pm 0.41^{\mathrm{b}}$ & $5.86 \pm 0.41^{\mathrm{ab}}$ & $6.71 \pm 0.41^{\mathrm{a}}$ \\
& 42 & $12.00 \pm 0.41^{\mathrm{b}}$ & $13.86 \pm 0.41^{\mathrm{a}}$ & $12.86 \pm 0.41^{\mathrm{ab}}$ \\
Proventrículo & 22 & $5.43 \pm 0.46^{\mathrm{a}}$ & $5.29 \pm 0.46^{\mathrm{a}}$ & $6.00 \pm 0.46^{\mathrm{a}}$ \\
& 42 & $9.57 \pm 0.46^{\mathrm{b}}$ & $11.29 \pm 0.46^{\mathrm{a}}$ & $11.29 \pm 0.46^{\mathrm{a}}$ \\
Molleja & 22 & $22.14 \pm 1.20^{\mathrm{a}}$ & $22.00 \pm 1.20^{\mathrm{a}}$ & $24.00 \pm 1.20^{\mathrm{a}}$ \\
& 42 & $40.00 \pm 1.20^{\mathrm{a}}$ & $41.86 \pm 1.20^{\mathrm{a}}$ & $41.43 \pm 1.20^{\mathrm{a}}$ \\
\hline
\end{tabular}

\pm Error estándar de la media

a,b Promedios con letra diferente en una misma fila son estadísticamente diferentes $(p<0.05)$

T1: Control (0\%); T2: Harina de laritaco 5 kg/t (0.5\%); T3: Harina de laritaco 10 kg/t (1\%) 
Cuadro 5. Efecto de la inclusión de harina de laritaco (Vernonanthura patens) en la dieta sobre la longitud de los intestinos $(\mathrm{cm})$ de pollos broiler a los 22 y 42 días de edad ( $\mathrm{n}=7$ pollos por tratamiento)

\begin{tabular}{lcccc}
\hline $\begin{array}{l}\text { Órgano } \\
(\mathrm{cm})\end{array}$ & $\begin{array}{c}\text { Edad } \\
(\text { días })\end{array}$ & T1 & T2 & T3 \\
\hline Intestino delgado & 22 & $159.29 \pm 5.13^{\mathrm{ab}}$ & $156.14 \pm 5.13^{\mathrm{b}}$ & $173.14 \pm 5.13^{\mathrm{a}}$ \\
& 42 & $194.71 \pm 5.13^{\mathrm{b}}$ & $215.71 \pm 5.13^{\mathrm{a}}$ & $216.29 \pm 5.13^{\mathrm{a}}$ \\
Intestino grueso & 22 & $8.5714 \pm 0.33$ & $8.14 \pm 0.33$ & $9.21 \pm 0.33$ \\
& 42 & $12.87 \pm 0.33^{\mathrm{b}}$ & $14.79 \pm 0.33^{\mathrm{a}}$ & $14.29 \pm 0.33^{\mathrm{a}}$ \\
Ciego & 22 & $14.00 \pm 0.49^{\mathrm{b}}$ & $14.14 \pm 0.49^{\mathrm{b}}$ & $15.29 \pm 0.49^{\mathrm{a}}$ \\
& 42 & $18.86 \pm 0.49^{\mathrm{b}}$ & $21.43 \pm 0.49^{\mathrm{a}}$ & $20.36 \pm 0.49^{\mathrm{a}}$ \\
\hline
\end{tabular}

\pm Error estándar de la media

a,b Promedios con letra diferente en una misma fila son estadísticamente diferentes $(p<0.05)$

T1: Control (0\%); T2: Harina de laritaco 5 kg/t (0.5\%); T3: Harina de laritaco 10 kg/t (1\%)

Cuadro 6. Efecto de la inclusión de harina de laritaco (Vernonanthura patens) en la dieta sobre las vellosidades y criptas de Lieberkühn $(\mathrm{mm})$ de pollos broiler a los 42 días de edad ( $\mathrm{n}=7$ pollos por tratamiento)

\begin{tabular}{lccc}
\hline & T1 & T2 & T3 \\
\hline Altura vellosidad & $0.69 \pm 0.05$ & $0.63 \pm 0.04$ & $0.68 \pm 0.04$ \\
Ancho vellosidad & $0.23 \pm 0.02^{\mathrm{b}}$ & $0.31 \pm 0.02^{\mathrm{a}}$ & $0.24 \pm 0.02^{\mathrm{b}}$ \\
Profundidad cripta & $0.40 \pm 0.02^{\mathrm{a}}$ & $0.22 \pm 0.02^{\mathrm{b}}$ & $0.14 \pm 0.02^{\mathrm{c}}$ \\
\hline
\end{tabular}

\pm Error estándar de la media

$a, b, c$ Promedios con letra diferente en una misma fila son estadísticamente diferentes $(p<0.05)$

T1: Control (0\%); T2: Harina de laritaco 5 kg/t (0.5\%); T3: Harina de laritaco 10 kg/t (1\%)

cual indica que la inclusión de harina de laritaco no afecta estas variables. En este sentido, Gonzalez (2016) utilizando diversos probióticos y Shiva et al. (2012) utilizando aceite esencial de orégano (Origanum vulgare) y extracto de jengibre (Zingiber officinale) no encontraron diferencias en consumo de alimento, Trujillo (2015) tampoco observó diferencias en peso corporal al suministrar harina de eucalipto (Eucalyptus citriodora) y Ordoñez et al. (2018) tampoco registraron diferencias significativas en conversión alimenticia al incluir orégano en las dietas de pollos. No obstante, Ordoñez et al. (2018) y Ebrahimnezhad et al. (2014) reportaron menores consumo de alimento, Gonzalez (2016) reportó mejoras significativas en el peso corporal al incluir extractos y harinas de diversas plantas en las dietas de pollos de engorde. 


\section{Órganos del Paquete Visceral}

Las aves de T3 y T2 presentaron el mayor peso en el segmento esófago-buche a los 22 y 42 días, respectivamente, en comparación con el grupo control $(\mathrm{p}<0.05)$. De otra parte, los pesos del hígado, corazón y molleja fueron similares entre tratamientos (Cuadro 4). Estos resultados concuerdan con los estudios de Ubua et al. (2019), quienes adicionaron harina de hoja de neem (Azadirachta indica) en el alimento y con Ebrahimnezhad et al.(2014) al incluir el jengibre en las dietas de pollos sobre los pesos de órganos viscerales.

La longitud del intestino delgado, intestino grueso y ciegos fue mayor a los 42 días en los pollos que se incluyó harina de laritaco en la dieta en comparación con el grupo control (Cuadro 5; $\mathrm{p}<0.05$ ). Esto difiere de lo reportado por Vera (2015) quienes no encontraron diferencia en la longitud del intestino delgado y de los ciegos al incluir aceite de sacha inchi (Plukenetia volubilis) en la dieta de pollos de engorde.

\section{Vellosidades Intestinales y Criptas de Lieberkühn}

El ancho de las vellosidades intestinales fue mayor en las aves de T2 en comparación con las medidas registradas en $\mathrm{T} 1$ y $\mathrm{T} 3$ $(p<0.05)$; sin embargo, no hubo diferencia significativa entre tratamientos concerniente a la altura de las vellosidades (Cuadro 6).

La profundidad de las criptas fue significativamente afectada por la concentración de harina de laritaco en la dieta $(\mathrm{p}<0.05$; Cuadro 6). En forma similar, Shiva et al. (2012) y Madrid et al. (2018) encontraron menor profundidad de criptas y mayores tamaños de vellosidades en pollos que recibían aceite esencial de orégano, y con el estudio de Chávez et al. (2016) con inclusión de cepas probióticas.

\section{Conclusiones}

- No se encontró efecto de la harina de laritaco sobre los parámetros productivos en pollos de engorde, indicando que la forma y a las dosis en que se incluyó el compuesto fitogénico no afectó el crecimiento de las aves.

- La inclusión de harina de laritaco originó un aumento en la longitud de los segmentos del intestino, logrando que el pollo tenga un intestino con más capacidad de absorción, necesario para un mejor aprovechamiento de los nutrientes.

- La inclusión de harina de laritaco mostró un efecto positivo sobre las vellosidades (más anchas) y las criptas (menos profundas) de pollos de engorde, indicando una mayor integridad y salud intestinal.

\section{Literatura Citada}

1. Barrera-Barrera HM, RodríguezGonzález SP, Torres-Vidales G 2014. Efectos de la adición de ácido cítrico y un probiótico comercial en el agua de bebida, sobre la morfometría del duodeno y parámetros zootécnicos en pollo de engorde. Orinoquía 18: 52-62.

2. Chávez L, López A, Parra J. 2016. Crecimiento y desarrollo intestinal de aves de engorde alimentadas con cepas probióticas. Arch Zootec 65: 51-58. doi: 10.21071/az.v65i249.441

3. Cobb-Vantress. 2015. Suplemento informativo sobre rendimiento y nutrición de pollos de engorde cobb 500. Cobbvantress. [Internet]. Disponible en: https:/ /cobbstorage.blob.core.windows.net/ guides/ee5706d0-5d14-11e8-9602$256 \mathrm{ac} 3 \mathrm{ce} 03 \mathrm{~b} 1$

4. Ebrahimnezhad Y, Azarakhsh V, Salmanzadeh M. 2014. The effects of ginger root (Zingiber officiale) processed to different levels on growth 
performance, carcass characteristics and blood biochemistry parameters in broiler chickens. Acad Environ Life Sci 3: 203-208.

5. Gachet M, Lecaro J, Kaiser M, Brun $R$, Navarrete H, Munoz, R, Schühly W. 2010. Assessment of anti-protozoal activity of plants traditionally used in Ecuador in the treatment of leishmaniasis. J Ethnopharmacol 128: 184-197. doi: 10.1016/j.jep.2010.01.007

6. Gonzalez I. 2016. Evaluación de probióticos sobre los índices productivos y la morfometría de las vellosidades intestinales en pollos de engorde. Tesis de Médico Veterinario Zootecnista. Ambato, Ecuador: Univ. Técnica de Ambato. $56 \mathrm{p}$.

7. Jaramillo AH. 2012. Evaluación de la mezcla de un ácido orgánico y un prebiótico en los parámetros productivos y alométricos de pollos de engorde con alimentación controlada. Rev Colomb Cienc Anim 5: 52-66.

8. Kvist L, Aguirre Z, Sánchez O. 2006. Bosques montanos bajos occidentales en Ecuador y sus plantas útiles. Bot Econ Andes Cent 205-223.

9. Madrid T, López A, Parra J. 2018. La ingesta de aceite esencial de orégano (Lippia origanoides) mejora la morfología intestinal en broilers. Arch. Zootec 67: 470-476.

10. Manzano P, Orellana T, Miranda M, Abreu J, Ruíz O, Peralta E. 2013. Algunos parámetros farmacognósticos de Vernonanthura patens (Kunth) H. Rob. (Asteraceae) endémica de Ecuador. Rev Cubana Plant Med 18: 131-139.

11. Ordoñez E, Del Carpio P, Cayo I. 2018. Suplementación alimenticia con orégano (Origanum vulgare) y complejo enzimático en pollos de carne: I. Indicadores Productivos. Rev Inv Cult 7: 31-44. doi: 10.18050/ucv-hacer.v7i1.-1348

12. Plaza J, Gómez C, Fontana L, Gil A. 2014. Modulation of immunity and inflammatory gene expression in the gut, in inflammatory diseases of the gut and in the liver by probiotics. World J Gastroenterol 20: 15632-15649. doi: 10.3748/wjg.v20.i42.15632

13. Roofchaee A, Irani M, Ebrahimzadeh M, Akbari M. 2011. Effect of dietary oregano (Origanum vulgare L.) essential oil on growth performance, cecal microflora and serum antioxidant activity of broiler chickens. Afr J Biotechnol 10: 6177-6183.

14. Shiva C, Bernal S, Sauvain M, Caldas J, Kalinowski J, Falcón N, Rojas R. 2012. Evaluación del aceite esencial de orégano (Origanum vulgare) y extracto deshidratado de jengibre (Zingiber officinale) como potenciales promotores de crecimiento en pollos de engorde. Rev Inv Vet Perú 23: 160-170. doi: 10.15381/rivep.v23i2.896

15. Trujillo N. 2015. Utilización de eucalipto (Eucalyptus citriodora) como promotor del crecimiento en dietas para pollos de engorde. Tesis de Médico Veterinario Zootecnista. Ambato, Ecuador: Univ. Técnica de Ambato. $86 \mathrm{p}$.

16. Ubua J, Ozung P, Inagu P. 2019. Dietary inclusion of neem (Azadirachta indica) leaf meal can influence growth performance and carcass characteristics of broiler chickens. Asian J Biol Life Sci 12: 180-186. doi: 10.3923/ajbs.2019.180.186

17. Vera H. 2015. Efecto de tres niveles de aceite de sacha inchi Plukenetia volubilis en la dieta de pollos de engorde en la línea Cobb. Tesis de Ingeniero Zootecnista. Riobamba, Ecuador: Escuela Superior Politécnica de Chimborazo. $43 \mathrm{p}$.

18. Zea O, Vílchez, C. 2014. Efecto de la suplementación con fuentes de cobre sobre el comportamiento productivo, morfometría intestinal y nivel de cobre hepático en pollos de carne. Rev Inv Vet Perú 25: 16-28. doi: 10.15381/ rivep.v25i1.8464 